\title{
16 Bibliografía
}

\section{Corpus}

Ávila, Raúl (coord.) (1980). Mi libro de primero. Parte I. México: CONALITEG.

Ávila, Raúl (coord.) (1980). Mi libro de primero. Parte ll. México: CONALITEG.

Ávila, Raúl (coord.) (1980). Mi libro de primero. Recortable. Parte I. México: CONALITEG.

Ávila, Raúl (coord.) (1980). Mi libro de primero. Recortable. Parte II. México: CONALITEG.

Ávila, Raúl (coord.) (1994). Español Primer Grado Lecturas. México: CONALITEG.

Chapela Mendoza, Luz María (1993). Libro integrado Primer Grado. México: CONALITEG.

Chapela Mendoza, Luz María (1993). Libro integrado Primer Grado Recortable. México:

CONALITEG.

De Bravo Ahuja, Gloria (coord.) (1972). Español Primer Grado. México: CONALITEG.

De Bravo Ahuja, Gloria (coord.) (1972). Español Primer Grado Recortable. México: CONALITEG.

Delgado, Ana Laura \& González, Margarita (1997). Conoce nuestra Constitución. Libros de

Texto Gratuitos. México: CONALITEG, SEP.

Domínguez Aguirre, Carmen \& León González, Enriqueta (1960). Mi libro de primer año. México: CONALITEG.

Domínguez Aguirre, Carmen \& León González, Enriqueta (1960). Mi cuaderno de trabajo de primer año. México: CONALITEG.

Domínguez Aguirre, Carmen \& León González, Enriqueta (1961). Mi Libro y mi Cuaderno de Trabajo de Primer Año. México: CONALITEG.

Gómez Palacio, Margarita (dir.) (1997). Español Primer Grado Actividades. México: CONALITEG. Gómez Palacio, Margarita (dir.) (1997). Español Primer Grado Recortable. México: CONALITEG. Gómez Palacio, Margarita (dir.) (1997). Español Primer Grado Lecturas. México: CONALITEG. Hernández Ruiz, Aida \& Reyes González, Patricia (comp.) (1993). Español Primer Grado Recortable. México: CONALITEG.

Vásquez de Knauth, Josefina (coord.) (1972). Ciencias Sociales Primer Grado. México: CONALITEG.

Vásquez de Knauth, Josefina (coord.) (1975). Ciencias Naturales Ciencias Sociales Primer Grado. México: CONALITEG.

\section{Bases de datos electrónicas}

Catálogo histórico de libros de texto gratuitos 1960-1917. Disponible en: https://historico.co naliteg.gob.mx/.

\section{Estudios}

Aboites Aguilar, Jaime (1998). Crecimiento y crisis de la economía mexicana. En Carlos A. Rozo (coord.), La política macroeconómica en México. México: Siglo XXI Editores. 
Aguilar Rivera, José Antonio (2010). Las transfiguraciones de la identidad nacional. En

S. Loaeza \& J. F. Prud'homme (coords.), Los grandes problemas de México. XIV. Instituciones y procesos politicos (pp. 532-552). México: El Colegio de México.

Álvarez de Testa, Lilian (1992). Mexicanidad y libro de texto gratuito. México: Universidad Nacional Autónoma de México.

Apple, Michael W. (1993). Official knowledge. Democratic education in a conservative age. New York/London: Routledge.

Apple, Michael \& Weiss, Lois (1983). Ideology and practice in schooling. Philadelphia: Temple University Press.

Ávila, Raúl (2011). Los libros de texto gratuitos y mi integración personal. En R. Barriga Villanueva (ed.), Entre paradojas: $A 50$ años de los libros de texto gratuitos (pp. 385-400). México: El Colegio de México; SEP, CONALITEG.

Atkinson, Max \& Heritage, John (1984). Introduction. En M. Atkinson \& J. Heritage (eds.), Structures of social action. Studies in Conversation Analysis (pp. 1-16). Cambridge: Cambridge University Press.

Baker, Carolyn, \& Johnson, Greer (2000). Stories of courtship and marriage: Orientations in openings, Narrative Inquiry, 10 (1), 1-25.

Bakhtin, Mikhail (1981). Discourse in the novel. En M. Holquist (ed.), The Dialogic Imagination: Four Essays (pp. 259-422). Austin: University of Texas Press.

Bamberg, Michael (1997). Positioning between structure and performance, Journal of Narrative and Life History, 7, 335-342.

Bamberg, Michael (2004). 'We are young, responsible and male': Form and function of 'slutbashing' in the identity constructions in 15 -year-old males, Human Development, 47, 331-353.

Bamberg, Michael G. \& McCabe, Allyssa (1998). Editorial, Narrative Inquiry, 8 (1), iii-v.

Bamberg, Michael \& Georgakopoulou, Alexandra (2008). Small stories as a new perspective in narrative and identity analysis, Text and Talk, 28, 377-396.

Barcelona, Antonio (2005). The fundamental role of metonymy in cognition, meaning, communication and form. En: A. Baicchi, C. Broccias \& A. Sansó (eds.), Modelling thought and constructing meaning. Cognitive models in interaction (pp. 109-124). Milano: Frencoangeli.

Barcelona, Antonio (2016). La metonimia conceptual. En I. Ibarretxe-Antuñano \& J. Valenzuela (eds.), Lingüística cognitiva (pp. 123-146). Barcelona: Anthropos.

Barriga Villanueva, Rebeca (2011). Entre paradojas: $A 50$ años de los libros de texto gratuitos. México: El Colegio de México; SEP, CONALITEG.

Barthes, Roland (1977). Introduction to the Structural Analysis of Narratives. En S. Heath (trad.), Image, Music, Text (pp. 79-124). New York: Hill and Wang.

Bauman, Zygmunt (2005). Identidad. Buenos Aires: Losada.

Bell, Allan (1984). Language Style as Audience Design. En N. Coupland \& A. Jaworski (1997, eds.) Sociolinguistics: a Reader and Coursebook (pp. 240-50). New York: St Mattin's Press Inc.

Bell, Susan (2006). Becoming a mother after DES: Intensive mothering in spite of it all. En A. De Fina, D. Schiffrin, \& M. Bamberg (eds.), Discourse and identity (pp. 233-54). Cambridge: Cambridge University Press.

Benwell, Bethan \& Stokoe, Elizabeth (2006). Discourse and identity. Edinburgh: Edinburgh University Press. 
Bernal Cigarroa, Agustín (1982). Los Libros de Ciencias Naturales y los Programas de 1972 y 1978. En E. González Pedrero (coord.), Los libros de texto gratuitos (pp. 315-327). México: SEP-CONALITEG.

Bourdieu, Pierre (1977). Outline of a theory of practice. Cambridge: Cambridge University Press.

Brice Heath, Shirley (1972). La política del lenguaje en México: De la Colonia a la Nación. México: SEP, Instituto Nacional Indigenista, Colección Sepini.

Briggs, Charles L. (1986). Learning how to ask: A sociolinguistic appraisal of the role of the interview in social science research. Cambridge: Cambridge University Press.

Bruner, Jerome (1986). Actual Minds, Possible Worlds. Cambridge, MA: Harvard University Press.

Bruner, Jerome (1990). Acts of meaning. Cambridge, MA: Harvard University Press.

Bruner, Jerome (1991). The Narrative Construction of Reality, Critical inquiry, 18 (1), 1-21.

Bucholtz, Mary (1999). You Da man: narrating the racial other in the production of White masculinity, Journal of Sociolinguistics, 3 (4), 46-479.

Bucholtz, Mary (2009). From Stance to Style: Gender, Interaction, and Indexicality in Mexican Immigrant Youth Slang. En A. Jaffe (ed.), Stance: Sociolinguistic Perspectives (pp. 146-170). Oxford / New York: Oxford University Press.

Bucholtz, Mary \& Hall, Kira (2005). Identity and interaction: a sociocultural linguistic approach. Discourse Studies, 7 (4-5), 585-614.

Cabanel, Patrick (2007). Le Tour de la nation par des enfants. Romans scolaires et espaces nationaux, $X I X-X X^{e}$ siècles. Paris: Belin.

Carr, David (1986). Time, Narrative, and History. Bloomington / Indianapolis: Indiana University Press.

Charteris-Black, J. (2004). Corpus approaches to critical metaphor analysis. Basingstoke: Palgrave Macmillan.

Chatman, Seymour (1990). Coming to terms. The rhetoric of narrative in fiction and film. Ithaca / London: Cornell University Press.

Chilton, Paul (2004). Analysing political discourse: theory and practice. London: Routledge.

Chilton, Paul (2005). Missing links in mainstream CDA: Modules, blends and the critical instinct. En R. Wodak \& P. Chilton (eds.), A New Agenda in (Critical) Discourse Analysis. Theory, methodology and interdisciplinarity (pp. 19-52). Amsterdam / Philadelphia: John Benjamins.

Croft, William (2002). The role of domains in the interpretations of metaphors and metonymies. En R. Dirven, \& R. Pörings (eds.), Metaphor and metonymy in comparison and contrast (pp. 335-371). Berlin / New York: Mouton Gruyter.

Croft, William \& Cruse, D. Alan (2004). Cognitive Linguistics. Cambridge: Cambridge University Press.

Culler, Jonathan (1980). Foreword En G. Genette: Narrative Discourse. An Essay in Method (pp. 7-14). Ithaca / New York: Cornell University Press.

Davies, Bronwyn. \& Harré, Rom (1990). Positioning: The discursive construction of selves, Journal for the Theory of Social Behavior, 20, 43-63.

De Fina, Anna (2003). Identity in Narrative: A Study of Immigrant Discourse. Philadelphia: John Benjamins.

De Fina, Anna (2006). Group identity, narrative and self-representations. En A. De Fina, D. Schiffrin \& M. Bamberg (eds.), Discourse and Identity (pp. 351-375). New York: Cambridge University Press. 
De Fina, Anna (2009). Narratives in interviews - The case of accounts. For an interactional approach to narratives genres, Narrative Inquiry, 19 (2), 233-258.

De Fina, Anna (2011). Researcher and Informant Roles in Narrative Interactions: Constructions of Belonging and Foreign-ness, Language in Society, 40, 27-38.

De Fina, Anna (2013a). Narratives as practices: negotiating identities though storytelling. En G. Barkhuizen (ed.), Narrative Research in Applied Linguistics (pp. 154-175). Cambridge University Press.

De Fina, Anna (2013b). Positioning level 3. Connecting local identity displays to macro social processes, Narrative Inquiry, 23 (1), 40-61.

De Fina, Anna (2017). Narratives and Politics. En R. Wodak \& B. Forchtner (eds.), Routledge Handbook of Language and Politics (pp. 233-246). London: Routledge.

De Fina, Anna, Schiffrin, Deborah \& Bamberg, Michael (eds.) (2006). Discourse and Identity. Cambridge: Cambridge University Press.

De Fina, Anna \& Georgakopolou Alexandra (2008). Introduction: Narrative analysis in the shift from texts to practices, Text \& Talk, 28 (3), 275-281.

De Fina, Anna \& Georgakopolou Alexandra (2012). Analyzing Narrative. Discourse and Sociolinguistic Perspectives. Cambridge: Cambridge University Press.

De Fina, Anna \& Georgakopolou Alexandra (2015). The handbook of narrative analysis. Somerset, US: Wiley-Blackwell.

De Fina, Anna \& Perrino Sabina (2011). Introduction: Interviews vs. 'natural' contexts: A false dilemma, Language in Society, 40, 1-11.

Deppermann, Arnulf (2015). Positioning. En A. De Fina \& A. Georgakopolou (eds.), The handbook of narrative analysis (pp. 369-387). Somerset, US: Wiley-Blackwell.

Dezheng, Feng (2011). Visual space and ideology. A critical cognitive analysis of spatial orientations in advertising. En O'Halloran, K. L. \& Smith, B.A. (eds.), Multimodal Studies. Exploring Issues and Domains (pp. 55-75). New York: Routledge.

Dirven, René (2005). Major strands in Cognitive Linguistics. En A. Baicchi, C. Broccias \& A. Sansó (eds.), Modelling thought and constructing meaning. Cognitive models in interaction (pp. 11-40). Milano: Frencoangeli.

Dirven, René, Frank, Roslyn \& Pütz, Martin (2003). Introduction: Categories, cognitive models and ideologies. En R. Dirven, R. Frank \& M. Putz (eds.), Cognitive models in language and thought: Ideology, metaphors and meanings (pp. 1-24). Berlin: Mouton de Gruyter.

Dirven, René, Polzenhagen, F. \& Wolf, H.G. (2007). Cognitive linguistics, ideology and critical discourse analysis. En D. Geeraerts \& H. Cuckyens (eds.), The Oxford Handbook of Cognitive Linguistics (pp. 1222-1240). Oxford: Oxford University Press.

Edwards, Derek (1997). Structure and function in the analysis of everyday narratives. En M. Bamberg (ed.), Oral versions of personal experience: Three decades of narrative analysis. Journal of Narrative and Life History, 7 (1-4), 335-342.

ELAN (Version 5.7) [Computer software]. (2019). Nijmegen: Max Planck Institute for Psycholinguistics, The Language Archive. Retrieved from https://archive.mpi.nl/tla/elan.

Elliott, Jane (2005). Using Narrative in Social Research. London: SAGE Publications.

Evans, Vyvyan (2007). A glossary of Cognitive Linguistics. Edinburgh: Edinburgh University Press.

Evans, Vyvyan \& Green, Melanie (2006). Cognitive Linguistics. An introduction. Edinburgh: Edinburgh University.

Fairclough, Norman (1989). Language and power. New York: Longman.

Fairclough, Norman (1992). Discourse and social change. Cambridge: Polity. 
Fairclough, Norman (1995). Critical discourse analysis: The critical study of language. London: Longman.

Fairclough, Norman \& Wodak, Ruth (1997). Critical discourse analysis. En T. van Dijk (ed.), Discourse as social interaction. Discourse studies: A multidisciplinary introduction. Vol 2. (pp. 258-284). London: Sage.

Farr, Robert M. \& Moscovici, Serge (eds.) (1984). Social representations. Cambridge: Cambridge University Press.

Fauconnier, Gilles (1994 [1985]). Mental spaces. Cambridge: Cambridge University Press.

Fauconnier, Gilles (1997). Mappings in thought and language. Cambridge: Cambridge University Press.

Fauconnier, Gilles \& Turner, Mark (2002). The way we think. Conceptual blending and the mind's hidden complexities. New York: Basics Books.

Fauconnier, Gilles \& Turner, Mark (2006 [1998]). Conceptual integration networks.

En D. Geeraerts (ed.), Cognitive Linguistics: Basic readings (pp. 303-371). Berlin: Mouton de Gruyter.

Favela Fierro, María Teresa (2009): La patria, raíces de México en los libros de texto, Discurso visual Revista digital: http://discursovisual.net/dvweb13/agora/agomaria.htm (01.08.17).

Fillmore, Charles (1977). Scenes-and-Frames Semantics. En A. Zampolli (ed.), Linguistic structures processing (pp. 55-81). Amsterdam: North Holland.

Fillmore, Charles (1985). Frames and the Semantics of Understanding, Quaderni di semantica, 6, 222-254.

Fillmore, Charles (2006). Frames Semantics. En D. Geeraerts (ed.), Cognitive Linguistics: Basic Readings (pp. 373-400). Berlin / New York: De Gruyter.

Fiske, Susan \& Taylor, Shelley (1991). Social cognition. New York: McGraw-Hill.

Flowerdew, John \& Richardson, John E. (eds.) (2018). The Routledge Handbook of Critical Discourse Studies. London / New York: Taylor \& Francis.

Fludernik, Monika (1996). Towards a 'natural' narratology. London / New York: Routledge. Fludernik, Monika (2003). Nartural narratology and cognitive parameters. En D. Herman (ed.), Narrative theory and the cognitive sciences (pp. 243-267). Stanford, CA: Center for the Study of Language and Information.

Foucault, Michael (1972). The archeology of knowledge. New York: Harper and Row.

Foucault, Michael (1979). Discipline and punish. New York: Vintage.

Foucault, Michael (1998). Historia de la Sexualidad I. La voluntad de saber. Madrid: Siglo XXI.

Fowler, Roger (1991). Language in the news: Discourse and ideology in the press. London: Routledge.

Fowler, Roger, Hodge, Robert, Kress, Gunther \& Trew, Tony (1979). Language and control. London: Routledge \& Kegan Paul.

Genette, Gérard (1980). Narrative discourse. An essay in method. J. E. Lewin (trad.). Ithaca / New York: Cornell University Press.

Georgakopoulou, Alexandra (2006). Thinking big with small stories in narrative and identity analysis, Narrative inquiry, 16 (1), 129-137.

Georgakopoulou, Alexandra (2015). Small Stories Research. Methods - Analysis - Outreach. En A. De Fina \& A. Georgakopoulou (eds.), The Handbook of Narrative Analysis (pp. 255-271). West Sussex: Wiley Blackwell.

Giles, Howard, Coupland, Nikolas \& Coupland, Justine (1991). Accommodation theory: Communication, context, and consequence. En H. Giles, J. Coupland, \& N. Coupland (eds.), 
Studies in emotion and social interaction. Contexts of accommodation: Developments in applied sociolinguistics (p. 1-68). Cambridge: Cambridge University Press.

Gilbert, Dennis (2003). Emiliano Zapata: Textbook Hero, Mexican Studies / Estudios Mexicanos, 19 (1), 127-159.

González Pedrero, Enrique (coord.) (1982). Los libros de texto gratuitos. México: SEPCONALITEG.

Goodwin, Charles (1984). Notes on story structure and the organization of participation. En M. Atkinson \& J. Heritage (eds.), Structures of Social Action. Studies in Conversation Analysis (pp. 225-246). Cambridge: Cambridge University Press.

Goodwin, Marjorie H. (1997). Towards families of stories in context. En M. Bamberg (ed.), Oral versions of personal experience: Three decades of narrative analysis. Journal of Narrative and Life History, 7 (1-4), 107-112.

Grady, Josef, Oakley, Todd. \& Coulson, Seana (1999). Blending and metaphor. En G. Steen \& R. Gibbs (eds.), Metaphor in cognitive linguistics. Philadelphia: John Benjamins.

Gramsci, Antonio (1930). Quaderni dal carcere. Disponible en http://quaderni.gramsciproject. org (01.04.19).

Greaves Laine, Cecilia (2001). Política educativa y libros de texto gratuitos. Una polémica en torno al control por la educación, Revista Mexicana de Investigación Educativa, 6 (12), mayo-agosto, $\mathrm{s} / \mathrm{n}$.

Guevara Niebla, Gilberto (1978). Antecedentes y desarrollo del movimiento de 1968. En C. Pereyra, A. Sánchez Rebolledo \& N. Espresate (eds.), Cuadernos Políticos, 17, 1-49.

Guevara Niebla, Gilberto (1988). La democracia en la calle: crónica del movimiento estudiantil mexicano. México: Siglo XXI.

Gutiérrez, Natividad (1998). Arquetipos y estereotipos en la construcción de la identidad nacional de México, Revista Mexicana de Sociología, 60 (1), 81-90.

Halliday, Michael Alexander Kirkwood (1973). Explorations in the Functions of Language. London: Edward Arnold.

Halliday, Michael Alexander Kirkwood (1978). Language as Social Semiotic: The Social Interpretation of Language and Meaning. London: Edward Arnold.

Hart, Christopher (2008). Critical discourse analysis and metaphor: Toward a theoretical framework, Critical Discourse Studies, 5, 91-106.

Hart, Christopher (2010). Critical Discourse Analysis and Cognitive Science. New perspectives on immigration discourse. Hamshire: Palgrave Macmillan.

Hart, Christopher (2011). Moving beyond metaphor in the Cognitive Linguistic approach to CDA. Construal operations in immigration discourse. En C. Hart (ed.), Critical Discourse Studies in Context and Cognition (pp. 171-192). Amsterdam / Philadelphia: John Benjamins.

Hart, Christopher \& Lukeš, Dominik (2010). Cognitive Linguistics in Critical Discourse Analysis. Application and theory. New Castle: Cambridge Scholars Publishing.

Haynes, John (1989). Introducing stylistics. London: Hyman.

Herman, David (2002). Story logic: Problems and possibilities of narratives. Lincoln / London: University of Nebraska Press.

Herman, David (ed.) (2003). Narrative Theory and the Cognitive Sciences. Standford, CA: CSL Publications.

Herman, David (2009). Basic elements of narrative. Lincoln / London: University of Nebraska Press. 
Herman, David, Jahn, Manfred \& Ryan, Marie-Laure (eds.) (2005), Routledge Encyclopedia of Narrative Theory. London / New York: Routledge.

Herman, Luc \& Vervaeck Bart (2005). Handbook of narrative analysis. Lincoln / London: University of Nebraska Press.

Hernández, Bertha (2011). "Así eran mis libros . . . "La colección pictórica de la Comisión Nacional de Libros de Texto Gratuitos. México: CONALITEG.

Hodge, Robert \& Kress, Gunther (1988). Social semiotics. Cambridge: Polity Press / Blackwell Publishers.

Hyvärinen, Matti (2006). Towards a conceptual history of narrative. En M. Hyvärinen,

A. Korhonen \& J. Mykkänen: The travel Concept of Narrative (pp. 20-40). Helsinki: Helsinki Collegium of Advance Studies. Consultado en https://helda.helsinki.fi/bitstream/handle/ 10138/25742/001_04_hyvarinen.pdf?sequence=1 (13.03.17).

Ibarretxe-Antuñano, Iraide (1999). Polysemy and metaphor in perception verbs: a crosslinguistic study (tesis doctoral). University of Edinburgh.

Jaso García, Sofía (1982). Los Libros de Español y los Programas de 1972 y 1978. En E. González Pedrero (coord.), Los libros de texto gratuitos (pp. 261-290). México: SEPCONALITEG.

Jefferson, Gail (1978). Sequencial aspects of storytelling in conversation. En J. Schenkein (ed.), Studies in the organization of conversation (pp. 79-112). New York: Academic Press.

Johnson, Greer (2008). Making visible an ideological dilemma in an interview narrative about social trauma, Narrative Inquiry, 18 (2), 187-205.

Kiesling, Scott (2006). Hegemonic identity-making in narrative. En A. De Fina, D. Shiffrin \& M. Bamberg (eds.), Discourse and identity (pp. 261-287). Cambridge University Press.

Koller, Veronika (2004). Metaphor and gender in business media discourse: A critical cognitive study. Basingstoke: Palgrave Macmillan.

Koller, Veronika (2005). Critical discourse analysis and social cognition: evidence from business media discourse, Discourse and Society, 16 (2), 199-224.

Koller, Veronika (2014). Cognitive Linguistics and Ideology. En J. Littlemore \& J. R. Taylor (eds.), The bloomsbury companion to cognitive linguistics (pp. 234-252). London: Bloomsbury.

Kövecses, Zoltan (2002). Metaphor: A Practical Introduction. New York: Oxford University Press.

Koven, Michele (2011). Comparing stories told in sociolinguistic interviews and spontaneous conversation, Language in Society, 40, 75-89.

Kress, Gunther (1985). Linguistic processes in sociocultural practice. Victoria: Deakin University Press,

Kress, Gunther (1989). Linguistics processes in sociocultural practice, $2^{\circ}$ ed. Oxford: Oxford University Press.

Kress, Gunther \& van Leewen, Theo (2006 [1996]). Reading images. The grammar of visual design. New York: Routledge.

Labov, William (1972). The transformation of experience in narrative syntax. En Language in the inner city. Studies in the Black English Vernacular (pp. 354-396). Philadelphia: University of Pennsylvania Press.

Labov, William \& Waletzky, Joshua (1967). Narrative analysis. En J. Helm (ed.), Essays on the Verbal and Visual Arts (pp. 12-44). Seattle: University of Washington Press.

Lakoff, George (1987). Women, fire and dangerous things: what categories reveal about the mind. Chicago: University of Chicago Press. 
Lakoff, George (2006). Conceptual metaphor. The contemporary theory of metaphor. En

D. Geeraerts (ed.), Cognitive Linguistics: Basic Readings. Berlin / New York: Mouton de Gruyter.

Lakoff, George \& Johnson, Mark (1986). Metáforas de la vida cotidiana. Madrid: Cátedra.

Lakoff, George \& Turner, Mark (1989). More than cool reason: a field guide to poetic metaphor.

Chicago: University of Chicago Press.

Langacker, Ronald (1987). Foundations of cognitive grammar: Teoretical prerequisites (Vol. 1).

Stanford: Stanford University Press.

Langacker, Ronald (2008). Cognitive grammar: a basic introduction. Oxford / New York: Oxford University Press.

Latapí, Pablo (1987). Análisis de un sexenio de educación en México 1970-1976. México: Editorial Nueva Imagen.

Latapí, Pablo (2004). La SEP por dentro. México: Fondo de Cultura Económica (Educación y pedagogía).

Ledin, Per \& Machin, David (2018). Multi-modal critical discourse analysis. En J. Flowerdew \&

J. E. Richardson (eds.), The Routledge Handbook of Critical Discourse Analysis (pp. 60-76). New York: Routledge.

Lerner, Victoria (12.03.1993). Nuevas luces; vieja polémica. Zona Abierta, suplemento de El Financiero, México, p. 41.

Lucius-Hoene, Gabriele \& Deppermann, Arnulf (2000). Narrative identity empiricized: A dialogical and positioning approach to autobiographical research interviews. Narrative Inquiry, 10 (1), 199-222.

Machin, David \& Mayr, Andrew (2012). How to do Critical Discourse Analysis. London: Sage. MacIntyre, Alasdair (1984). After Virtue: A Study in Moral Theory. Notre Dame: University of Notre Dame Press.

Maldonado, Ricardo (2012). La gramática cognitiva. En I. Ibarretxe-Antuñano \& J. Valenzuela (dirs.), Lingüística Cognitiva (pp. 213-247). Barcelona: Anthropos.

Maldonado, Ricardo (1999). Espacios mentales y la interpretación del se impersonal. En

A. Vigueras, (ed.), El centro de Lingüística Hispánica y la lengua española en Conmemoración de los 30 años de la creación del Centro de Lingüística Hispánica (pp. 205-228). México: Instituto de Investigaciones Filológicas, UNAM.

Mandler, Jean \& Johnson, Nancy (1977). Remembrance of things parsed: story structures and recall, Cognitive Psicology, 9, 111-151.

McAdams, Dan P. (1988). Power, Intimacy, and the Life Story: Personological Inquiries into Identity. New York / London: The Guilford Press.

Merino Huerta, Mauricio (1982). Los libros de Ciencias Sociales y los Programas de 1972 y 1978. En E. González Pedrero (coord.), Los libros de texto gratuitos (pp. 315-327). México: SEP-CONALITEG.

Mink, Louis O. (1987). Historical Understanding. Ithaca / London: Cornell University Press.

Mishler, Elliot G. (1986). Research interviewing: Context and narrative. Boston: Harvard University Press.

Monsiváis, Carlos (2003 [1979]). La ofensiva ideológica de la derecha. En: P. González Casanova \& E. Florescano (coords.), México hoy (pp. 306-328). México: Siglo XXI Editores. Moscovici, Serge (1981). L'Âge des foules : un traité historique de psychologie des masses. Paris: Fayard. 
Moscovici, Serge (1984). The phenomenon of social representations. En R. M. Farr \&

S. Moscovici (eds.), Social representations (pp. 3-69). Cambridge: Cambridge University Press.

Narvaja de Arnoux, Elvira (2008). Los discursos sobre la nación y el lenguaje en la formación del Estado (Chile, 1842-1862) Estudio glotopolítico. Buenos Aires: Santiago Arcos Editor. Narvaja de Arnoux, Elvira \& del Valle, José (2010). Las representaciones ideológicas del lenguaje. Discurso político y panispánico, Spanish in Context, 7 (1), 1-24.

Nealson, Jeffrey (2008). Foucault beyond Foucault. Stanford University Press.

O’Halloran, Kieran (2003). Critical Discourse Analysis and language cognition. Edinburgh: Edinburgh University Press.

Ochs, Elinor (1992). Indexing gender. En A. Duranti \& C. Goodwin (eds.), Rethinking context: Language as an interactive phenomenon (pp. 335-358). Cambridge: Cambridge University Press.

Ochs, Elinor \& Capps, Lisa (2001). Living Narrative: Creating Lives in Everyday Storytelling. Cambridge, MA: Harvard University Press.

Prince, Gerald (2003 [1987]). A Dictionary of Narratology. Lincoln / London: University of Nebraska Press.

Reisigl, Martin (2011). Pragmatics and (Critical) Discourse Analysis - commonalities and differences. En C. Hart (ed.), Critical Discourse Studies in Context and Cognitio (pp. 7-26). Amsterdam, Philadelphia: John Benjamins.

Reisigl, Martin \& Wodak, Ruth (2001). Discourse and discrimination. Rethorics of racism and antisemitism. London: Routledge.

Riessman Kohler, Catherine (1991). Beyond reductionism: Narrative genres in divorce accounts. Journal of Narrative and Life History, 1, 41-68.

Riessman Kohler, Catherine (1993). Narrative analysis. Newbury Park, CA: Sage.

Riessman Kohler, Catherine (2008). Narrative methods for the human sciences. Thousand Oaks, CA: Sage.

Rosch, Eleanor (1978). Principles of Categorization. En E. Rosch \& B. Lloyd (eds.), Cognition and Categorization (pp. 27-48). Hillsdale, N): Lawrence Erlbaum.

Rosch, Eleanor \& Mervis, Carolyn (1975). Family Resemblances: Studies in the Internal Structure of Categories, Cognitive Psychology, 7 (4), 573-605.

Ruiz de Mendoza, F. \& Otal Campos, J. (2002). Metonymy, grammar and communication, Granada: Comares.

Rumelhart, David (1975). Notes of a schema for stories. En D. Bobrow \& A. Collins (eds.), Representation and understanding (pp. 211-236). New York: Academic Press.

Rumelhart, David (1977). Understanding and summarizing brief stories. En D. LaBerge \& J. Samuels (eds.), Basic processes in reading: perception and comprehension (pp. 265-303). Hillsdale, N): Erlbaum.

Ryan, Marie-Laure (1991). Possible worlds, artificial intelligence and narrative theory. Bloomington: Indiana University Press.

Ryan, Marie-Laure (2005). Narrative. En D. Herman, M. Jahn \& M.L. Ryan (eds.), Routledge Encyclopedia of Narrative Theory (pp. 344-348). London / New York: Routledge.

Sacks, Harvey (1974). An analysis of the course of a joke's telling in conversation. En R. Bauman \& J. Sherzer (eds.), Explorations in the Ethnography of Speaking (pp. 337-353). Cambridge: Cambridge University Press.

Salcedo Aquino, Roberto (1982). El desarrollo de los Libros de Texto Gratuitos. En E. González Pedrero (coord.), Los libros de texto gratuitos (pp. 31-46). México: SEP-CONALITEG. 
Sarbin, Theodore Roy (1986). Narrative psychology: The storied nature of human conduct. New York: Praeger.

Schank, Roger C. \& Abelson, Roger P. (1977). Scripts, Plans, Goals and Understending. Erlbaum: Hillsdale.

Schank, Roger C. \& Abelson, Roger P. (1987). Guiones, Planes, Metas y Entendimiento. Barcelona: Paidós.

Schegloff, Emanuel (1997). Narrative analysis 30 years later. En M. Bamberg (ed.), Oral versions of personal experience: Three decades of narrative analysis. Journal of Narrative and Life History, 7 (1-4), 97-106.

Schiffrin, Deborah (1987). Discourse markers (No. 5). Cambridge: Cambridge University Press.

Shapira, Yoram (1978). La política exterior de México bajo el régimen de Echeverría: Retrospectiva, Foro Internacional XIX, 1 (73), 62-91.

Silverstein, Michael (1976). Shifters, linguistic categories, and cultural description. En K. H. Basso \& H. A. Selby (eds.), Meaning in anthropology (pp. 11-55). Albuquerque: University of New Mexico Press.

Silverstein, Michael (1979). Language structure and linguistic ideology. En P. R. Clyne, W. F. Hanks \& C. L. Hofbauer (eds.), The elements: A parasession on linguistic units and levels (pp. 193-248). Chicago, IL: Chicago Linguistic Society / University of Chicago.

Stein, Nancy \& Glenn, Christine (1979). An analysis of story comprehension in elementary school children. En R. Freedle (ed.), New directions in discourse processing (pp. 53-120). Norwood, NJ: Ablex.

Strawson, Galen (2004). Against narrativity, Ratio, 17 (4), 428-452.

Tajfel, Henri (1981). Human Groups and Social Categories: Studies in Social Psychology. Cambridge: Cambridge University Press.

Tajfel, Henri \& Turner, John (1986). An Integrative Theory of Intergroup Conflict. En W. G. Austin \& S. Worchel (eds.), The Social Psicology of Intergroup Relations (pp.33-53). California: Brooks / Cole Publishing Company.

Talmy, Leonard (1977). Rubber Sheet Cognition in Language. En W. a. Beach et al. (eds.), Papers of the Thirteenth Regional Meeting Chicago Linguistic Society (pp. 612-628). Chicago: Chicago Linguistic Society.

Talmy, Leonard (1978). The Relations of Grammar to Cognition: A Synopsis. En D. Waltz (ed.), Proceedings of TINLAP-2: Theoretical Issues in Natural Language Processing (pp. 612-628). Urbana: University of Illinois Coordinated Science Laboratory.

Talmy, Leonard (1983). How language structures space. En L. Hebert, Jr. Pick \& L. P. Acredolo (eds.), Spatial orientation: theory, research and application (pp. 225-282). New York: Plenum Press.

Talmy, Leonard (1988). The Relations of Grammar to Cognition. En B. Rudzka-Ostyn (ed.), Topics in Cognitive Linguistics (pp. 165-205). Amsterdam: John Benjamins.

Talmy, Leonard (2000). Toward a cognitive semantics, Concept structuring systems (Vol. 1). Cambridge: Cambridge University Press.

Taylor, Charles (1989). Sources of the Self: The making of modern identity. Cambridge MA: Harvard University Press.

Taylor, Donald M. \& Moghaddam, Fathali M. (1994). Theories of intergroup relations. International social psichological perspectives. Westport, CT / London: Praeger Publishers.

Taylor, John. R. (1999 [1995]). La categorizzazione linguistica. (S. Giannini, trad.). Macerata: Quodliber. 
Thorndyke, Perry (1977). Cognitive structures in comprehension and memory on narrative discourse, Cognitive Psicology, 9, 77-110.

Todorov, Tzvetan (1969). Grammaire du Décaméron. La Hague: Mouton.

Tomasello Michael. (2005 [1999]). Le origini culturali della cognizione umana. (M. Riccucci, trad.). Bologna: Il Mulino.

Torres Barreto, Arturo (2007). Los libros de texto gratuitos de historia en la política educativa de México 1959-1994 (tesis de doctorado en historia). Universidad Nacional Autónoma de México.

van Dijk, Teun (1985). Cognitive situation models in discourse production: The expression of ethnic situations in prejudiced discourse. En J. P. Forgas (ed.), Language and social situations (pp. 61-79). New York: Springer.

van Dijk, Teun (1993). Modelos en la memoria. El papel de las representaciones de la situación en el proceso del discurso, Revista Latina de Pensamiento y Lenguaje, 2 (1), 39-55.

van Dijk, Teun (1997). Discurso, cognición y sociedad. Signos. Teoría y práctica de la educación, 66-74.

van Dijk, Teun (1995). Discourse analysis as ideology analysis. En C. Schäffner \& A. Wenden (eds.), Language and Peace (pp. 17-33). Aldershot: Dartmouth Publishin.

van Dijk, Teun (1998): Ideology. A multidisciplinary approach. London, Thousand Oaks, New Delhi: SAGE Publications.

van Dijk, Teun (2001). Critical discourse analysis. En D. Schiffrin, D. Tannen \& H. E. Hamilton (eds.), The handbook of discourse analysis (pp. 352-371). Oxford: Blackwell.

van Dijk, Teun (2002). Political discourse and political cognition. En P. A. Chilton \&

C. Schäffner (eds.), Politics as Text and Talk. Analytical approaches to political discourse (pp. 204-236). Amsterdam: John Benjamins.

van Dijk, Teun (2003). Ideología y discurso. Barcelona: Ariel Lingüística.

van Dijk, Teun (2008). Discourse and context. A sociocognitive approach. Cambridge:

Cambridge University Press.

van Dijk, Teun (2009). Critical discourse studies: A sociocognitive approach. En R. Wodak

\& M. Meyer (eds.), Methods of critical discourse analysis (pp. 62-86). London: Sage.

van Dijk, Teun (2011). Ideología y discurso. Barcelona: Ariel Lingüística.

van Dijk, Teun \& Kintsch, Walter (1983). Strategies of discourse comprehension. New York: Academic Press.

Villa Lever, Lorenza (s/a): La Historia en los Libros de Texto Gratuitos. 50 años y cuatro concepciones. Consultado en https://www.academia.edu/4077509/DEFLa_Historia_en_ los_Libros_de_Texto_Gratuitos?auto=download (18.07.19).

Villa Lever, Lorenza (1988). Los libros de texto gratuitos. La disputa por la educación en México. México: Universidad de Guadalajara.

Villa Lever, Lorenza (2011). Reformas educativas y libros de textos gratuitos. En R. Barriga

Villanueva (ed.), Entre paradojas: A 50 años de los libros de texto gratuitos (pp. 159-177).

México: El Colegio de México / SEP, CONALITEG.

Wenger, Etienne, McDermott, Richard, Snyder, William (2002). Cultivating communities of practice: a guide to managing knowledge. Boston, MA: Harvard Business School Press.

Werth, Paul (1999). Text worlds: Representing conceptual space in discourse. London: Longman.

White, Hayden (1981). The Value of Narrativity in the Representation of Reality. En

W. J. T. Mitchell (ed.), On Narrative (pp. 1-23). Chicago: University of Chicago Press.

Wittgenstein, Ludwig (1953). Philosophische Untersuchungen. London: Macmillan. 
Wodak, Ruth (1996). Disorders of discourse. London: Longman.

Wodak, Ruth (2001). The discourse-historical approach. En R. Wodak \& M. Meyer (eds.), Methods of Critical Discourse Analysis (pp. 63-94). London: SAGE.

Wodak, Ruth \& Meyer, Michael (2016). Methods of critical discourse studies. London: Sage.

Wortham, Stanton (2000). Interactional positioning and narrative self-construction.

En M. Bamberg \& A. McCabe (eds.), Narrative Inquiry, 10 (1), 157-198.

Wortham, Stanton (2001). Narratives in action. New York: Teachers College, Columbia University.

Wortham, Stanton, Mortimer, Katherine, Lee, Kathy, Allard, Elaine \& White, Kimberly (2011). Interviews as interactional data, Language in Society, 40, 39-50. 